\title{
A postal survey of GDPs views on referrals to a local secondary care service in restorative dentistry
}

\author{
Perceptions of general dental practitioners of a local secondary care service in restorative dentistry $\quad$ K. J. Fairbrother \\ and F. S. A. Nohl BrDent J 2000; 188: 99-102
}

\begin{abstract}
Aim
A major role of the hospital based secondary care service in restorative dentistry is to accept referrals in order to formulate treatment plans. The aim of this survey was to improve service provision at Newcastle Dental Hospital by establishing baseline quality perceptions from referring general dental practitioners (GDPs).
\end{abstract}

\section{Method \\ A postal questionnaire was sent to 393 randomly selected local GDPs, with a response rate of $67 \%$.}

\section{Results}

Results indicated great demand for advice and treatment which was higher than expected for temporomandibular joint problems. $42 \%$ of GDPs felt that treatment plans were not helpful and the period of waiting for a response following consultation too long. The reasons for this perception are discussed.

\section{Conclusions}

The need for better communication between primary and secondary care was highlighted, as was the distinct preference of GDPs for hospital consultants rather than registered specialist practitioners to carry out specialist treatment.

\section{In brief}

- This paper highlights the need for an accessible and comprehensive secondary care service in restorative dentistry.

- It emphasises the need for quality in the secondary care service in restorative dentistry.

- There is also a need for improved communication between hospital specialists and practitioners.

- Pertinent issues are raised about the usefulness of treatment plans returned to practitioners.

\section{Comment}

A number of reports in recent years have highlighted the need for specialist advice and treatment for patients presenting with a range of clinical problems within the field of restorative dentistry. A large proportion of these referrals results in advice on possible treatment plans being sent to the referring practitioners, but little attention has been directed in previous studies to ascertaining the views of the practitioners on the advice received.

The authors have conducted a survey of general dental practitioners practising within the areas of the former FHSAs of North Tyneside, Northumberland, Newcastle, South Tyneside/Gateshead, Durham, and Sunderland, to seek their views on the consultant service in restorative dentistry provided within the Newcastle Dental Hospital.
The vast majority of respondents reported that they referred patients at some time for advice and/or treatment, with an average frequency of one referral per year. Referrals were spread across the range of restorative disciplines with a high expectation of treatment rather than advice in relation to periodontics, prosthodontics and endodontics. The management of temporomandibular joint problems also generated a high volume of referrals, with relatively fewer practitioners seeking advice about implants.

The majority of practitioners felt that the treatment proposals which they received were helpful and were likely to be achievable within the constraints of general dental practice. A substantial minority however felt that the treatment plans proposed were unrealistic and some found them difficult to understand or accomplish.

Very few dentists had practice guidelines for referral and this is perhaps an area where there is scope for collaboration between the consultants and the referring practitioners. Further postgraduate courses might help to address the difficulties perceived by some dentists in undertaking the treatment proposed by the consultants. The authors are to be commended for undertaking this study which is very much in tune with current trends towards quality control and clinical governance and the establishment of outcome measures.

\footnotetext{
J Ralph

Consultant in Restorative Dentistry,

Leeds Dental Institute
} 